\title{
Heating the corona by nanoflares: simulations of energy release triggered by a kink instability
}

\author{
P. K. Browning ${ }^{1}$, C. Gerrard ${ }^{2}$, A. W. Hood ${ }^{2}$, R. Kevis ${ }^{2}$, and R. A. M. Van der Linden ${ }^{3}$ \\ 1 Jodrell Bank Centre for Astrophysics, School of Physics and Astronomy, University of Manchester, Manchester M13 9PL, UK \\ e-mail: p.Browning@manchester.ac.uk \\ 2 School of Mathematics and Statistics, University of St Andrews, St Andrews, Fife KY16 9SS, UK \\ 3 SIDC, Royal Observatory of Belgium, Ringlaan 3, 1180 Brussels, Belgium
}

Received 5 December 2007 / Accepted 11 April 2008

\begin{abstract}
Context. The heating of solar coronal plasma to millions of degrees is likely to be due to the superposition of many small energyreleasing events, known as nanoflares. Nanoflares dissipate magnetic energy through magnetic reconnection.

Aims. A model has been recently proposed in which nanoflare-like heating naturally arises, with a sequence of dissipation events of various magnitudes. It is proposed that heating is triggered by the onset of ideal instability, with energy release occurring in the nonlinear phase due to fast magnetic reconnection. The aim is to use numerical simulations to investigate this heating process.

Methods. Three-dimensional magnetohydrodynamic numerical simulations of energy release are presented for a cylindrical coronal loop model. Initial equilibrium magnetic-field profiles are chosen to be linearly unstable, with a two-layer parameterisation of the current profile. The results are compared with calculations of linear instability, with line-tying, which are extended to account for a potential field layer surrounding the loop. The energy release is also compared with predictions that the field relaxes to a state of minimum magnetic energy with conserved magnetic helicity (a constant $\alpha$ force-free field).

Results. The loop initially develops a helical kink, whose structure and growth rate are generally in accordance with linear stability theory, and subsequently a current sheet forms. During this phase, there is a burst of kinetic energy while the magnetic energy decays. A new relaxed equilibrium is established that corresponds quite closely to a constant $\alpha$ field. The fraction of stored magnetic energy released depends strongly on the initial current profile, which agrees with the predictions of relaxation theory.

Conclusions. Energy dissipation events in a coronal loop are triggered by the onset of ideal kink instability. Magnetic energy is dissipated, leading to large or small heating events according to the initial current profile.
\end{abstract}

Key words. Sun: corona - Sun: magnetic fields - magnetohydrodynamics (MHD) - plasmas

\section{Introduction}

It is a major challenge in astrophysics to explain how the coronae of the Sun and other stars are heated to temperatures in the millions of degrees (e.g. Browning 1991; Narain \& Ulmscheider 1996; Mandrini et al. 2000; Klimchuck 2006). Although it is widely accepted that the heating mechanism depends on the coronal magnetic field, the details of the process remain controversial. The "nanoflare" scenario (Parker 1988) has been widely studied; it proposes that coronal heating is due to the superposition of many small - and as yet observationally undetectable - flare-like energy-releasing events. The underlying energy-release mechanism in coronal heating is thus the same as in large flares, namely magnetic reconnection (e.g. Priest \& Forbes 2002). Magnetic reconnection converts stored magnetic energy into thermal energy and particle kinetic energy on timescales that are much more rapid than global ohmic diffusion. According to the nanoflare scenario, coronal heating is a sporadic rather than smooth, continuous process; this is compatible with many observations from space-borne observatories, which indicate that transient energy releasing events are widespread in the solar atmosphere. However, it has not been possible to determine conclusively whether the occurrence rate of nanoflares is sufficiently high for them to provide the energy required to heat the corona (Aschwanden 1999; Parnell 2000; Benz 2002;
Aschwanden \& Parnell 2002). Indeed, in the foreseeable future it may be difficult if not impossible to resolve this purely on observational grounds. It is thus of considerable interest to be able to predict a priori how frequently energy-releasing events of different sizes occur and how much energy is released at the different scales, and this is one of the key aims of the present work. Here we build on a new coronal heating model proposed by Browning \& Van der Linden (2003) which allows the distribution of heating-events (nanoflares and larger events) to be calculated ab initio.

The overall magnetic energy-release in many localised magnetic reconnection events, as expected to occur in complex coronal magnetic field configurations, is difficult to calculate directly. One fruitful approach is to use magnetic relaxation theory, originally developed for laboratory plasmas by Taylor (1974) but since applied extensively to the solar corona (e.g. Heyvaerts \& Priest 1984; Browning \& Priest 1986; Browning et al. 1986; Browning 1988; Vekstein et al. 1993; Kusano \& Nishikawa 1996; Lothian \& Browning 2000; Zhang \& Low 2003; Melrose 2004; Priest et al. 2005). In an ideal plasma, the magnetic helicity, defined as

$K=\int_{V} \boldsymbol{A} \cdot \boldsymbol{B} \mathrm{d} V$ 
is conserved. Here, $\boldsymbol{B}$ is the magnetic field and $\boldsymbol{A}$ the vector potential, so that $\boldsymbol{B}=\nabla \times \boldsymbol{A}$. When the volume of interest has magnetic flux crossing the boundaries, as is the case for solar coronal loops since field lines interlink the photosphere, the relative helicity must be used since the simple definition above is not unique (gauge dependent). We use the convenient formulation of Finn \& Antonsen (1985).

Relaxation theory postulates that in a highly conducting plasma, in the presence of small-scale magnetic reconnection, the global magnetic helicity survives as an invariant, whereas magnetic energy is dissipated by reconnection. In other words, helicity dissipation is much weaker than energy dissipation (Berger 1984; Taylor 1986); a simple justification for this, which will be useful in interpreting the results presented later, is as follows (Browning 1988). It may be easily shown from the MHD equations that the resistive dissipation rates of magnetic energy and helicity are

$$
\frac{\mathrm{d} K}{\mathrm{~d} t}=-2 \int \eta(\boldsymbol{j} \cdot \boldsymbol{B}) \mathrm{d} V, \quad \frac{\mathrm{d} W}{\mathrm{~d} t}=-\int \eta(\boldsymbol{j} \cdot \boldsymbol{j}) \mathrm{d} V,
$$

where $W$ is the magnetic energy, $\boldsymbol{j}=(\nabla \times \boldsymbol{B}) / \mu_{0}$ the current density, $\mu_{0}$ the magnetic permittivity and $\eta$ the resistivity. Assume that dissipation occurs mainly in thin current layers of width $l$, with $l \ll L$ (where $L$ is the global length scale), characteristic of magnetic reconnection, so that $j \sim \frac{B}{\mu_{0} l}$. Hence

$$
\frac{\mathrm{d} K}{\mathrm{~d} t} \sim-\left(2 \eta B^{2} L^{3}\right) /\left(\mu_{0} l\right), \quad \frac{\mathrm{d} W}{\mathrm{~d} t} \sim-\left(\eta B^{2} L^{3}\right) /\left(\mu_{0}^{2} l^{2}\right),
$$

so that

$$
\frac{(1 / K) \mathrm{d} K / \mathrm{d} t}{(1 / W) \mathrm{d} W / \mathrm{d} t} \sim \frac{l}{L} \ll 1 .
$$

Thus the dissipation of helicity is much slower than that of energy. Note that here we have neglected driving terms, which inject both energy and helicity through boundaries. Helicity injection due to photospheric footpoint motions can indeed be very important in the solar corona (e.g. Heyvaerts \& Priest 1984; Berger 1984), but is not discussed further here as it is absent in the model problem that is numerically simulated here, although helicity (and energy) are injected during the slow evolution leading to the establishment of the unstable equilibrium (Browning \& Van der Linden 2003). For a more detailed discussion of the role of helicity in magnetic reconnection, see Ji (1998).

If a magnetic field is disrupted, it tends to relax towards the state of minimum magnetic energy subject to the constraint of helicity conservation. This minimum energy or relaxed state is the well known linear or constant- $\alpha$ force-free field

$\nabla \times \boldsymbol{B}=\alpha \boldsymbol{B}$,

where

$\alpha=\frac{\mu_{0} j_{/ /}}{B}$,

(Taylor 1974). Such relaxation has recently been directly observed in the solar corona (Nandy et al. 2003). Any energy difference between the initial field and the relaxed state is usually assumed to be dissipated as heat. During the relaxation process, some magnetic energy might initially be converted into kinetic energy, but this will subsequently be dissipated by viscosity or other processes (this is implicit in relaxation theory since the relaxed state is static).

In order to apply these ideas to solar coronal heating, consider that the coronal magnetic field is stressed by photospheric footpoint motions whose timescale is slow compared with an Alfvén travel time along the field lines. The field thus evolves, in general, into a nonlinear force-free state, with some excess magnetic energy. If this is disrupted in some way, it will then relax to the lower energy constant- $\alpha$ force-free field, releasing any free energy as heat (Heyvaerts \& Priest 1984). The cycle of energy build-up and relaxation repeats, giving heating by a stress-and-relax process. One of the difficulties of most previous relaxation-based coronal heating models was that the energy release depends on the relative time-scales of the relaxation and driving processes. This leaves a question: for how long does energy build-up as the field is stressed by footpoint motions, before a heating-event occurs? Relaxation theory itself cannot answer this question. An answer was suggested by Browning \& Van der Linden (2003), who proposed that a heating-event is triggered by the onset of ideal MHD instability.

The reason for choosing ideal, rather than resistive, instabilities is that, in very highly conducting coronal plasma, only ideal instabilities have sufficiently rapid timescales to be relevant to flare onset or coronal heating. Naively, it might seem that ideal instabilities intrinsically cannot dissipate energy and are thus irrelevant as part of a heating process. However, there are clear indications from 3D MHD simulations in cylindrical loop models that in its nonlinear phase, the ideal kink instability generates strong current sheets in which fast, driven magnetic reconnection dissipates energy rapidly in the presence of small (but nonvanishing) resistivity (e.g. Baty \& Heyvaerts 1996; Velli et al. 1997; Arber et al. 1999; Baty 2000). Plasma flows generated by the growing kink mode act as a driver for reconnection, creating current sheets and pushing together oppositely directed fields. It is likely that the time-scale of this reconnection process will scale as the Alfvén time. Indeed, reconnection in the nonlinear phase of an ideal kink has been proposed as a flare mechanism, and it is a natural consequence of the nanoflare hypothesis to propose the same mechanism for coronal heating.

The coronal heating process proposed by Browning \& Van der Linden (2003) is thus as follows. The coronal field is stressed by photospheric footpoint motions, evolving quasistatically through a sequence of equilibria until it becomes ideally unstable. At this point, a dynamic heating-event ensues, at the end of which the field attains the minimum energy or constant- $\alpha$ state, with the same helicity but lower energy. The heat released during the event may be calculated as the difference between the energies of the stressed state and the relaxed state - which is always a positive quantity, since the latter is a minimum energy state. The value of $\alpha$ for the relaxed state is determined by the helicity conservation constraint. The process is investigated quantitatively for a simple model problem consisting of a cylindrical coronal loop magnetic-field of length $L$ and radius $R$, with the photosphere represented by boundaries at the ends of the cylinder $z=0, L$. The initial nonlinear force-free equilibrium, established by slow rotations of the photospheric footpoints, is given by a two-layer model with a piece-wise constant profile of $\alpha$. Thus, we have

$\nabla \times \boldsymbol{B}=\alpha(r) \boldsymbol{B}$,

where

$\alpha=\alpha_{1}, r<R_{\mathrm{c}}, \alpha=\alpha_{2}, r>R_{\mathrm{c}}$.

The field components are continuous across the layer boundary $r=R_{\mathrm{c}}$ although the current is discontinuous there. This formulation of the initial force-free state provides a convenient twoparameter family, which can approximate to a wide range of current profiles (Melrose et al. 1994). The linear stability threshold 


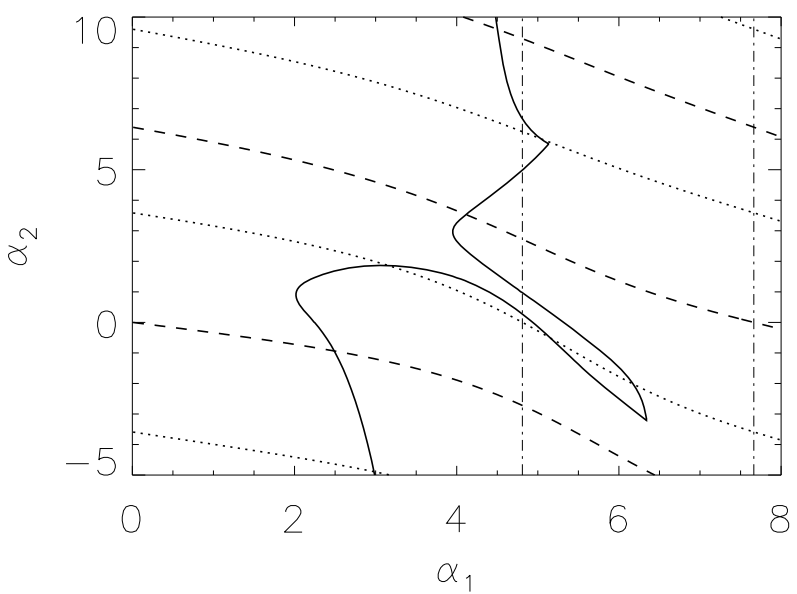

Fig. 1. The marginal stability boundary (solid line) for the ideal $m=$ 1 kink mode in the $\left(\alpha_{1}, \alpha_{2}\right)$ plane as calculated with the CILTS code. Here we used $R_{\mathrm{c}}=0.5$, aspect ratio $L / R=20$ and a rigid wall boundary at the edge of the loop $(r=R=1)$. The stable region is to the left of the line. The dotted and dashed curves show the successive zeroes at $r=R$ of $B_{z}$ and $B_{\theta}$, respectively, while the vertical dashed lines indicate for which values of $\alpha_{1}$ either of the field components are zero at the transition point $r=R_{\mathrm{c}}$.

for ideal $m=1$ kink instability was calculated using the CILTS code (see Van der Linden \& Hood 1999 for details about the method), and is reproduced here in Fig. 1.

For each point on the marginal stability curve, the energy release during helicity-conserving relaxation to a constant- $\alpha$ state (a point on the diagonal of Fig. 1) is determined. It is found that, for the main branch of the stability curve, heating-events of a wide range of sizes are obtained, ranging over about 3 ordersof-magnitude. The energy release may be usefully expressed

$\delta W=\frac{1}{\mu_{0}} \psi_{0}^{2} \frac{L}{R^{2}} \delta W^{*}=\frac{\pi^{2} L R^{2} B^{2}}{\mu_{0}} \delta W^{*}$,

where $\psi_{0}$ is the loop flux, $B$ a typical magnetic field and $\delta W^{*}$ is the dimensionless energy per unit length, which varies from about 0.001 to 1 depending on where the marginal stability curve is crossed. It is expected that the process will repeat: thus, the relaxed equilibrium will be stressed by the footpoint motions, evolving again quasi-statically until the instability threshold is again crossed and another heating-event ensues. Thus, coronal heating proceeds by a series of discrete transient heating-events or "nanoflares". The precise distribution of event sizes depends on the driving motions: but it is clear that the stability curve is most likely to be crossed at or near its nose, corresponding to a small heating-event, whereas larger events will be rarer.

The primary aim of the present paper is to compare the results of the Browning \& Van der Linden (2003) model, which uses relaxation theory to determine the energy release, with the results of a 3D numerical simulation solving the full nonlinear MHD equations. However, this investigation should not be seen merely as a test of relaxation theory, with the code used for verification (or otherwise): the numerical code itself also has limitations, notably it is unable to access the very large magnetic Reynold's numbers and correspondingly very wide range of length-scales, from current sheet width to global scales, that are expected to be found in the real solar corona. On the other hand, the numerical simulations do provide valuable information about the dynamics of relaxation which is beyond the scope of relaxation theory.
In performing this comparison, several aspects of coronal physics are elucidated, which are of intrinsic interest and relevance. Firstly, we improve the ideal linear stability map of a line-tied solar coronal loop calculated earlier, by relaxing the effects of the outer conducting wall. Secondly, better understanding is obtained of the nonlinear kink instability, for a range of current profiles. Thirdly, the effects on the energy available by relaxation of a range of current profiles and boundary positions are explored.

The details of the method and the model problem are outlined in Sect. 2. Extensions to the theory, both the linear stability results and the relaxation model, including an investigation of varying the boundary position, are presented in Sect. 3. The results of the numerical simulation are presented in Sect. 4, and compared both with the predictions of linear stability theory and with relaxation theory as appropriate. Finally, conclusions are outlined in Sect. 5.

\section{Numerical details and initial equilibria}

We carry out the nonlinear simulations using a 3D MHD Lagrangian-remap code, Lare3d, which is described fully in Arber et al. (2001). The code solves the MHD equations given by,

$$
\begin{aligned}
\frac{\partial \rho}{\partial t} & =-\nabla \cdot(\rho \boldsymbol{v}), \\
\frac{\partial}{\partial t}(\rho \boldsymbol{v}) & =-\nabla \cdot(\rho \boldsymbol{v} \boldsymbol{v})+\frac{1}{\mu_{0}}(\nabla \times \boldsymbol{B}) \times \boldsymbol{B}-\nabla P, \\
\frac{\partial \boldsymbol{B}}{\partial t} & =\nabla \times(\boldsymbol{v} \times \boldsymbol{B})-\nabla \times\left(\eta \frac{\nabla \times \boldsymbol{B}}{\mu_{0}}\right), \\
\frac{\partial}{\partial t}(\rho \epsilon) & =-\nabla \cdot(\rho \boldsymbol{\epsilon})-P \nabla \cdot \boldsymbol{v}+\eta j^{2}
\end{aligned}
$$

with specific energy density,

$\epsilon=\frac{P}{(\gamma-1) \rho}$,

where $v$ is the velocity, $P$ the thermal pressure, $\gamma=5 / 3$ the ratio of specific heats and $\rho$ the mass density. We ignore the effects of gravity, thermal conduction, radiation and heating.

The equations are made dimensionless by setting

$\boldsymbol{r} \longrightarrow r^{*} \tilde{\boldsymbol{r}}, \quad \boldsymbol{B} \longrightarrow B^{*} \tilde{\boldsymbol{B}}, \quad \boldsymbol{v} \longrightarrow v_{\mathrm{A}} \tilde{\boldsymbol{v}}$

$P \longrightarrow P^{*} \tilde{P}, \quad t \longrightarrow t^{*} \tilde{t}, \quad \rho \longrightarrow \rho^{*} \tilde{\rho}$,

where a tilde denotes a dimensionless variable. $v_{\mathrm{A}}$ is the Alfvén speed given by $v_{\mathrm{A}}=B^{*} / \sqrt{\mu_{0} \rho^{*}}, t^{*}=r^{*} / v_{\mathrm{A}}$ is the Alfvén transit time, $P^{*}=B^{* 2} / \mu_{0}$. Here, $r^{*}=R$, the loop radius, and $B^{*}=B_{1}$, the initial axial field at $r=0$. Thus, we obtain dimensionless equations and removing the tildes from the dimensionless quantities, they reduce to Eqs. (10)-(14) with $\mu_{0}=1.0$. The current is in units of $B_{0}^{*} / \mu_{0} r^{*}$. Since the resistivity which we will take is not uniform we keep a normalised $\eta$ explicitly in the equations by taking $\eta^{*}=\mu_{0} r^{*} v_{\mathrm{A}}$. The choice of the form of the resistivity follows Arber et al. (1999). We take, in dimensionless variables,

$\eta=\eta_{0} \operatorname{MAX}\left(0, \frac{|j|}{j_{\text {crit }}}-1\right)$

the critical current at which "anomalous resistivty" switches on is $j_{\text {crit }}=5$ (in dimensionless units)and $\eta_{0}=0.0001$; We limit the 

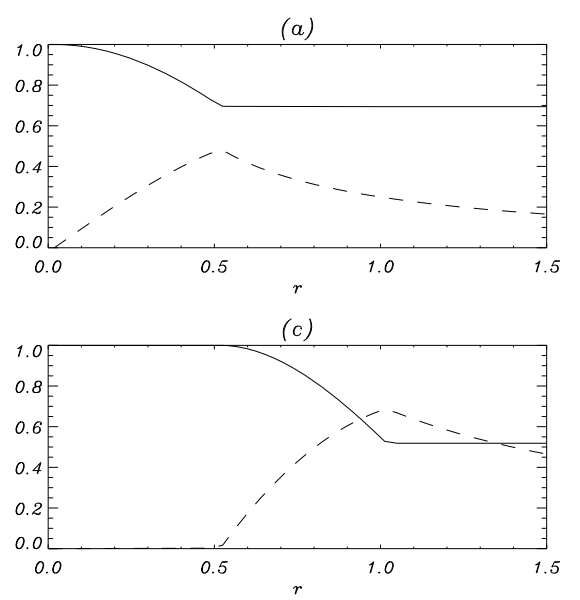

(e)

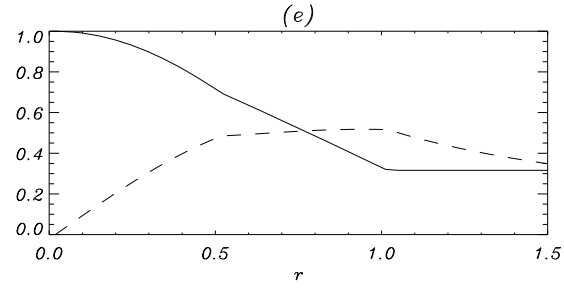

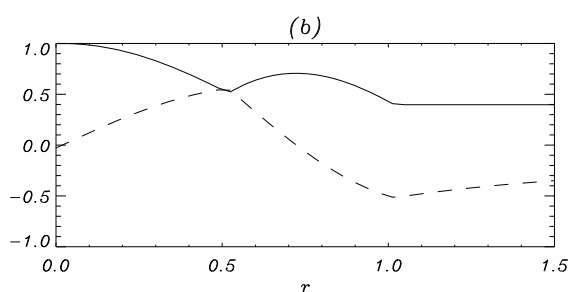
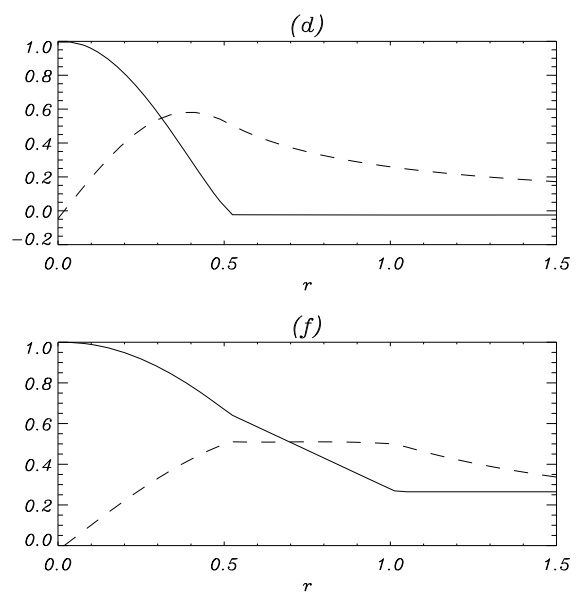

Fig. 2. Plots of $B_{z}$ (unbroken line) and $B_{\theta}$ (dashed line) against radius for cases 1 (a), 2 (b), 3 (c), 4 (d), 5 (e) and 6 (f). resistivity so that it cannot exceed $10^{-3}$, and there is no background resistivity.We calculate the relative helicity by making use of Eq. (2) above, which is directly integrated in time, with the initial value given by an exact analytical calculation for the helicity of the initial equilibrium (taking account of the external potential field, which contributes to the helicity through its interlinkage with loop field; with a square boundary corresponding to the numerical region).

The Lagrangian step is fully 3D, uses the predictor-corrector method and artificial viscosity. The remap step uses Van Leer gradient limiters (Van Leer 1997), applied to the density, specific energy density, velocities and magnetic fluxes to ensure that it is monotonicity preserving. Furthermore, Lare3d uses Evans and Hawley constrained transport (Evans \& Hawley 1988) to guarantee that if $\nabla \cdot \boldsymbol{B}$ is initially zero (which we ensure it is) it is maintained at zero to machine precision throughout the evolution. The numerical grid is staggered so that the density, pressure and specific energy density are defined at the cell centres; the velocities at the vertices; the magnetic field components at the cell faces and the current components along the edges of the numerical cell. $|j|=\left(j_{x}^{2}+j_{y}^{2}+j_{z}^{2}\right)^{1 / 2}$ and the resistivity are defined at the same vertices as the velocities. The staggered grid reduces the amount of averaging required in some of the calculations, thus reducing the associated error, and removes checkerboard biasing.

For each of the cases which we have investigated we have taken a computational domain with sizes $L_{x}=L_{y}=3$ and $L_{z}=20$. Each equilibrium is represented as a loop of radius 1 and, therefore, each loop has an aspect-ratio of 20, as in Browning \& Van der Linden (2003). We have chosen the size of the box in the $x$ - and $y$-directions such that the boundary conditions should not significantly affect the evolution of the loop. We impose line-tied boundary conditions in the $z$-direction. We run each simulation on an $81^{2} \times 160$ grid and carry out simulations without imposed resistivity and with imposed resistivity.
The initial field is taken to be in unstable equilibrium. The equilibria are defined as described in Browning \& Van der Linden (2003) and by Eq. (7) so that we have;

$B_{z}=B_{1} J_{0}\left(\alpha_{1} r\right), B_{\theta}=B_{1} J_{1}\left(\alpha_{1} r\right), \quad\left(r \leq R_{\mathrm{c}}\right)$,

$B_{z}=B_{2} J_{0}\left(\alpha_{2} r\right)+C_{2} Y_{0}\left(\alpha_{2} r\right)$,

$B_{\theta}=B_{2} J_{1}\left(\alpha_{2} r\right)+C_{2} Y_{1}\left(\alpha_{2} r\right), \quad\left(R_{\mathrm{c}}<r<1.0\right)$,

where

$C_{2}=B_{1} \frac{J_{0}\left(\alpha_{1} R_{\mathrm{c}}\right) J_{1}\left(\alpha_{2} R_{\mathrm{c}}\right)-J_{1}\left(\alpha_{1} R_{\mathrm{c}}\right) J_{0}\left(\alpha_{2} R_{\mathrm{c}}\right)}{\Delta}$

$B_{2}=B_{1} \frac{J_{1}\left(\alpha_{1} R_{\mathrm{c}}\right) Y_{0}\left(\alpha_{2} R_{\mathrm{c}}\right)-J_{0}\left(\alpha_{1} R_{\mathrm{c}}\right) Y_{1}\left(\alpha_{2} R_{\mathrm{c}}\right)}{\Delta}$,

and,

$\Delta=\frac{2}{\pi \alpha_{2} R_{\mathrm{c}}}$

We take $R_{\mathrm{c}}=0.5, B_{1}=1$ and in the rest of the box impose a potential field that matches the equilibrium field at $r=1.0$. Thus the initial fields are continuous throughout the numerical box, while the $\alpha$ profile is piecewise constant. The six cases that we have investigated are (see Fig. 2): (1) $\alpha_{1}=2.3$ and $\alpha_{2}=0.01$; (2) $\alpha_{1}=3.0$ and $\alpha_{2}=-3.5$; (3) $\alpha_{1}=0.01$ and $\alpha_{2}=2.3$; (4) $\alpha_{1}=$ 4.9 and $\alpha_{2}=0.01$; (5) $\alpha_{1}=2.3$ and $\alpha_{2}=1.5$; (6) $\alpha_{1}=2.5$ and $\alpha_{2}=1.69$; we focus most attention on the first three. These equilibria are chosen so that all are initially linearly unstable, but are either fairly close to the original marginal stability curve calculated in Browning \& Van der Linden (2003), reproduced in Fig. 1, or to the newly calculated stability threshold, described below. We apply a small velocity perturbation to trigger the kink instability and then investigate how the instability evolves and how the loop relaxes. 


\section{Linear stability and relaxed state calculations}

Before presenting the results of these $3 \mathrm{D}$ nonlinear numerical simulations, we first revisit the linear stability analysis of the equilibria described above. The results in Browning \& Van der Linden (2003) assumed a cylindrical conducting shell at the outer boundary of the loop $(r=1)$. However, the nonlinear code, being in Cartesian rather than cylindrical coordinates, has a rectangular outer conducting-shell at $x=y=0$ and $x=y=3$, with a potential field in the region between the "loop" (with centre located at $x=y=1.5$ and boundary at $r=1$ ) and the wall. As the linear stability code does not accommodate a square boundary, we consider instead a loop in $r<1$ surrounded by a potential field with an outer cylindrical conducting-wall at $r=R_{\mathrm{w}}>1$. By increasing $R_{\mathrm{w}}$ until its effects are insignificant, it should be possible to match the nonlinear simulation where the wall is at a sufficiently large distance that it has little effect at least in the initial stage of the development of the instability. Clearly this configuration must be less stable than the original model. It is also a better representation of the solar corona, since the effect of unrealistic conducting boundaries is weakened. The effect of the size of a potential field region surrounding a twisted forcefree loop has also been considered by Baty (2001).

The ideal linear stability of a loop with a surrounding potential field is studied using the CILTS code, which uses an iterative process to find the eigenvalue $\gamma^{2}$ of the linearised MHD equations. Figure 3 shows the growth-rate $\gamma$ for the cases studied in detail numerically (see next section), as a function of the wall distance $R_{\mathrm{w}}$. It is evident that the wall has little effect on the growth-rates (so that the stability is the same as if the wall were at infinite distance), for $R_{\mathrm{W}}>1.5$ and certainly for $R_{\mathrm{W}} \approx 3$. We thus calculate stability properties for $R_{\mathrm{W}}=3$ for comparison with the nonlinear simulations. Note that the square boundary of the the numerical simulations lies between the inscribed circles $R_{\mathrm{w}}=1.5$ and the circumscribed circle $R_{\mathrm{W}}=3$, so for detailed comparisons of growth-rates and so on, both extremes should be considered.

Unstable eigenfunctions for the current profiles studied are shown in Fig. 4. Although we used cylindrical coordinates in the calculations, the eigenfunctions have been transformed into cartesian coordinates for ease of comparison with the nonlinear simulations. We plot $V_{x}(x, z)$ along a plane cutting the loop along its axis. The linear eigenfunctions clearly show the different structures of the unstable modes in the equilibria selected. For cases 1 and 2 the eigenfunction is naturally confined to the non-potential loop field, which explains why the distance to the outer wall has virtually no influence on the growth-rate (see Fig. 3), even for small values of $R_{\mathrm{w}}$.

The marginal stability curve is found by starting with a known unstable point in $\left(\alpha_{1}, \alpha_{2}\right)$ parameter space, and then scanning until a first stable point is found. Due to symmetry, the boundary only needs to be calculated for positive $\alpha_{2}$ and the complete map is obtained by mirroring to get negative values. The results are shown in Fig. 5. It should be noted that, as expected, the stable region is smaller than the orginal stable region as calculated in Browning and Van der Linden (Fig. 1), and is fully contained within it. The first stable region in Fig. 5 is fully closed: in particular, the unstable "nose" in Fig. 1 and the stable region for large values of $\alpha_{2}$ near the $\alpha_{2}$ axis (which was actually a region of weak instability) have disappeared. The test cases for nonlinear simulation are marked on the diagram: it may be seen that, with the newly calculated stability boundary, some cases are now deeply within the unstable region. In order to facilitate the interpretation of the stability map, we have added

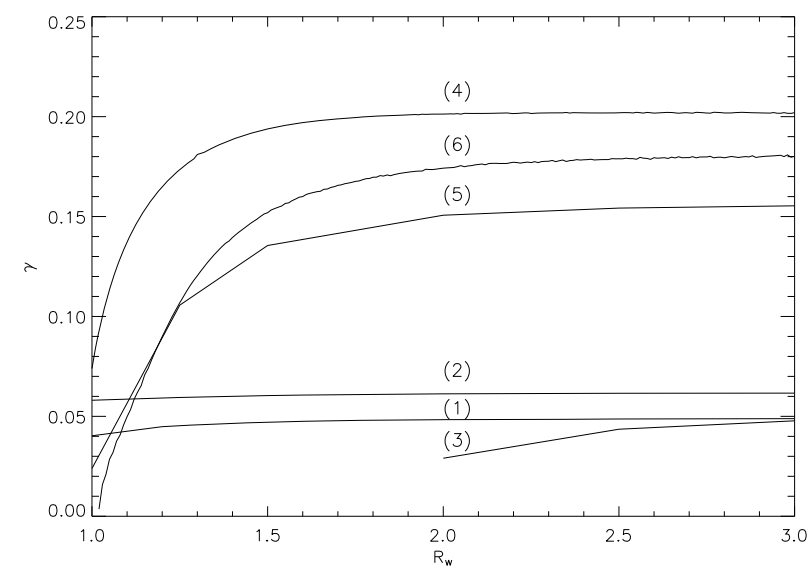

Fig. 3. Growth-rate $\gamma$ as a function of $R_{\mathrm{w}}$ for the six cases studied in this paper. Labels on the graphs correspond to the case number attributed in the text.
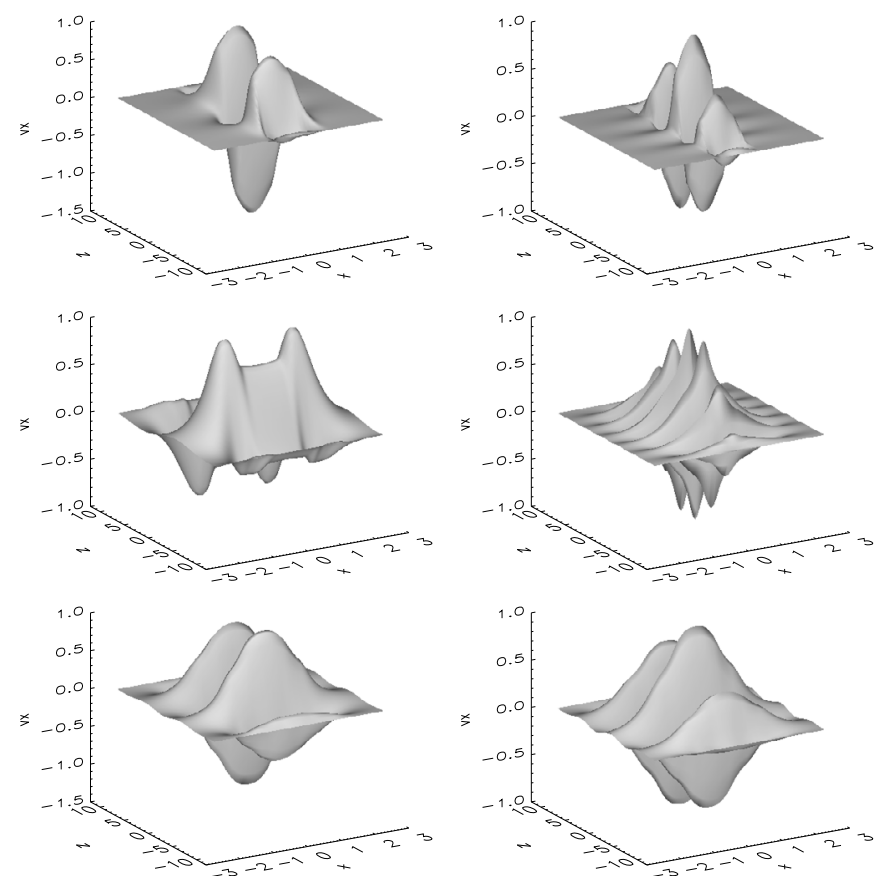

Fig. 4. Linear eigenfunctions for the six cases studied. We plot $V_{x}(x, z)$ along the plane $y=0$, which contains the cylinder axis $r=0$. The figures have been placed in the order of the case number.

curves showing reversals, within the loop radius $R$, of axial field $B_{z}$ (dashed lines) and of azimuthal field $B_{\theta}$ (dotted lines). It has been shown that the energy principle may be written such that the only destabilising term is proportional to $\alpha B_{z} B_{\theta}$ (De Bruyne $\&$ Hood 1992), so that crossing one of these reversal lines takes the equilibrium into a different stability regime.

The final $\alpha$ value and the energy release, according to relaxation theory, will also be affected by the addition of a potential field layer and the position of the external boundary. Thus, we re-calculate the energy difference between the initial field and the minimum energy constant- $\alpha$ field, including the effects of a potential field layer in the initial field between the loop boundary $r=R$ and the conducting wall $r=R_{\mathrm{w}}$. The relaxed state has a single value of $\alpha$ in the whole region $\left(r<R_{\mathrm{W}}\right)$; this value is calculated by noting that both helicity $K$ and total axial flux $\psi_{\mathrm{w}}=\psi\left(R_{\mathrm{w}}\right)$ are conserved during relaxation. Note that the helicity is also affected by the potential field layer surrounding the 


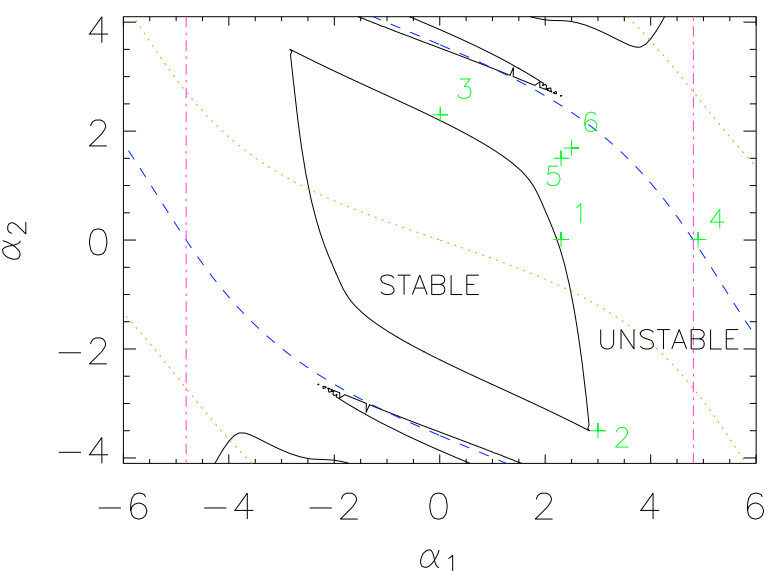

Fig. 5. Stability map for the same loop model as in Fig. 1, but now surrounded by a potential field bounded by a rigid wall at $R_{\mathrm{w}}=3$. Solid black curves mark the boundary between stable and unstable regions. The six cases studied in detail in this paper are also indicated.

loop: even though an isolated potential field has zero helicity, the potential field region contributes to the total helicity through the interlinkages of its fluxes with the loop fluxes. The energy release depends quite strongly on the wall position, both because the net volume is increased as the wall radius increases and because the relaxed state $\alpha$ value decreases: in fact, if the wall were moved to infinity, the field would relax to a potential state and all the free energy of the initial loop would be released. In order to compare with the nonlinear results, we take $R_{\mathrm{w}}=1.5$, giving a circle inscribed within the square numerical boundary (the missing "corner" regions are of small volume). In dimensionless units, taking (as in the nonlinear numerical simulations), the axial field on axis $B_{1}=1$, the energy release $\delta W$ per unit length is 0.0338 for $R_{\mathrm{W}}=1$ (no potential field region, as in previous work) and increases to 0.089 for $R_{\mathrm{W}}=1.5$, for the current profile of test case $1\left(\alpha_{1}=2.3, \alpha_{2}=0.01\right)$; the relaxed state $\alpha$ values are 1.124 (no potential field region) and 0.5703 (potential field out to $R_{\mathrm{W}}=1.5$.)

\section{Results}

We present results from the nonlinear numerical simulations for the six test cases, focussing on cases (1), (2) and (3), and compare with the linear stability results and relaxation theory. These three cases are close to the marginal stability curve with an outer potential layer, descibed in the previous section while the remaining cases are well inside the unstable region (but are close to the original stability curve shown in Fig. 1). This is reflected in the magnitude of the growth rates of the various cases.

\subsection{Case 1: $\alpha_{1}=2.3$ and $\alpha_{2}=0.01$}

Isosurfaces of current at successive times are shown in Fig. 6. The loop shows signs of growing instability by $t=100 \tau_{\mathrm{A}}$, where the loop can be seen to be kinked (Fig. 6, left). We estimate the growth-rate of the instability from the kinetic energy in the numerical results by taking $\mathrm{d}(\log (K . E)) / \mathrm{d} t$, (see Fig. 7). This gives us an estimate of $\gamma \approx 0.06$ for the initial linear instability, which agrees very well with the linear result which is likewise $\gamma \approx 0.05$ for high values of $R_{\mathrm{w}}$. The shape of the helical deformation nicely matches the linear eigenfunction (Fig. 4).

A current concentration starts to form at $t=110 \tau_{\mathrm{A}}$ at approximately $r=0.75$ and can be seen as a helical ribbon
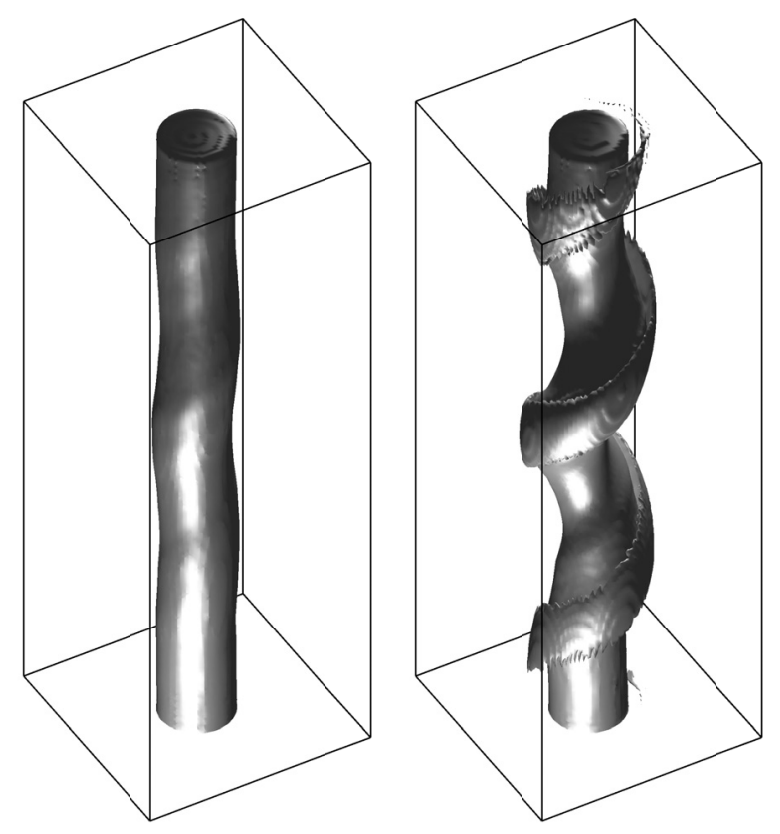

Fig. 6. Isosurfaces of current at $|j|=1.5$ at $t=100 \tau_{\mathrm{A}}$ (left hand side) and $t=124 \tau_{\mathrm{A}}$ (right hand side) for case (1).

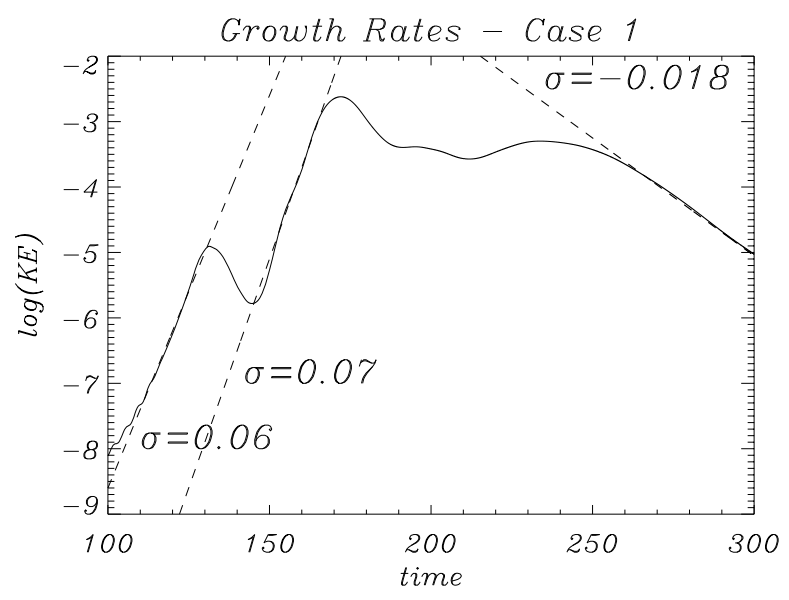

Fig. 7. Plot of log kinetic energy as a function of time and the estimated growth rates.

wrapped around the cylinder by $t=124 \tau_{\mathrm{A}}$ (Fig. 6, right). For this case we use the form of the resistivity discussed earlier with $j_{\text {crit }}=6$ and $\eta_{0}=1 \times 10^{-4}$. The current exceeds this critical value at approximately $t=130 \tau_{\mathrm{A}}$ and Fig. 8e shows that the ohmic heating increases from this time showing that the resistivity begins to affect the evolution of the loop.

As we have a high concentration of current in this period, a resistive plasma reconnection occurs. The effects of this can be seen clearly in the energy plots (Fig. 8). Just before $t=200 \tau_{\mathrm{A}}$ the magnetic energy begins to drop rapidly whilst the kinetic energy suddenly increases and then peaks and decreases more slowly. This behaviour is characteristic of reconnection, the kinetic energy increase being associated with fast reconnective outflows. Notice that there appear to be a series of reconnection episodes, with several peaks in kinetic energy of varying magnitudes. We continue the simulation until $t=400 \tau_{\mathrm{A}}$ by which time the magnetic energy is beginning to plateau, while the current is still decreasing and is dropping below the critical value for the resistivity to be triggered. The helicity (Fig. 8f) varies in 

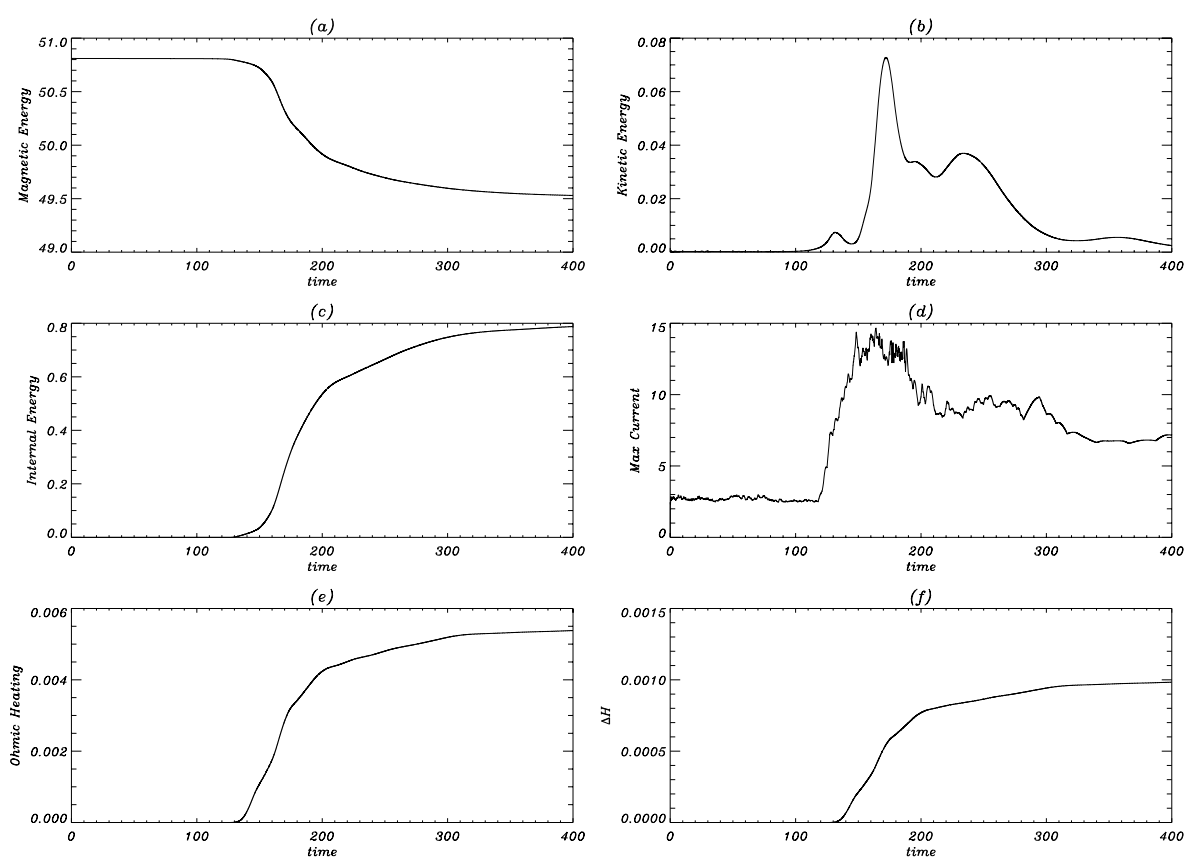

Fig. 8. Plots of a) magnetic energy, b) kinetic energy, c) internal energy, d) maximum current, e) Ohmic-heating and f) change in helicity as functions of time for case (1). The initial helicity, calculated analytically, is 24.935 . a similar manner to the ohmic heating but its variation is relatively weaker. The increase in helicity is due to the large negative current spikes (see Fig. 6), thus giving a positive change in $K$ according to Eq. (2).

We note that the relative change in helicity $(\delta K / K \sim$ $0.0005)$ is much less than the relative change in magnetic en$\operatorname{ergy}(\delta W / W \sim 0.03)$. This is entirely consistent with the underlying hypothesis of relaxation theory. Furthermore, from Eq. (4) we see that the change in helicity relative to the change in energy should scale like $l / L$; with the limited grid resolution available to the $3 \mathrm{D}$ code, this can be no less than $1 / 81$ in the present simulation, whereas we might expect $l / L \sim S^{-1 / 2}$, for example, which will be far smaller at large $S$ in the real corona. This shows that relaxation theory works best in very high magneticReynolds-number plasmas, which should indeed exist in the solar corona but which cannot be accessed by present day numerical simulations.

The cumulative ohmic-heating (Fig. 8e) is almost constant from $t=300 \tau_{\mathrm{A}}$ suggesting that the resistivity is too low to allow further reconnection or diffusion to take place except at isolated locations. On the other hand, the peak current and kinetic energy still appear to be decaying at this time, indicating that the relaxation process is not yet complete.

How close is the plasma to the final constant- $\alpha$ relaxed state? Relaxation theory predicts a constant- $\alpha$ solution, valid in the central part of the loop, of the form

$$
B_{z}=0.7801 J_{0}(0.5703 r), \quad B_{\theta}=0.7801 J_{1}(0.5703 r) .
$$

The value of $\alpha$ is determined by conservation of helicity and total axial flux from the initial state. Figure 9 shows the axial magnetic-field component, $B z$ as a function of $x$ at $y=0$ and $z=$ 0 at the final time of $t=400$. The long dashed curve is the initial axial field. The solid curve is the simulation result at $t=400$, while the dotted curve is the predicted constant $\alpha$ field based on relaxation theory with $\alpha=0.5703$ and $B_{z}(0,0,0)=0.78$. The short dashed curve shows the constant- $\alpha$ solution based on $\alpha=0.85$ and $B_{z}(0,0,0)=0.81$. This provides a good fit with the simulation result. However, it should be noted that the axis has been displaced by a distance of approximately -0.2 . This
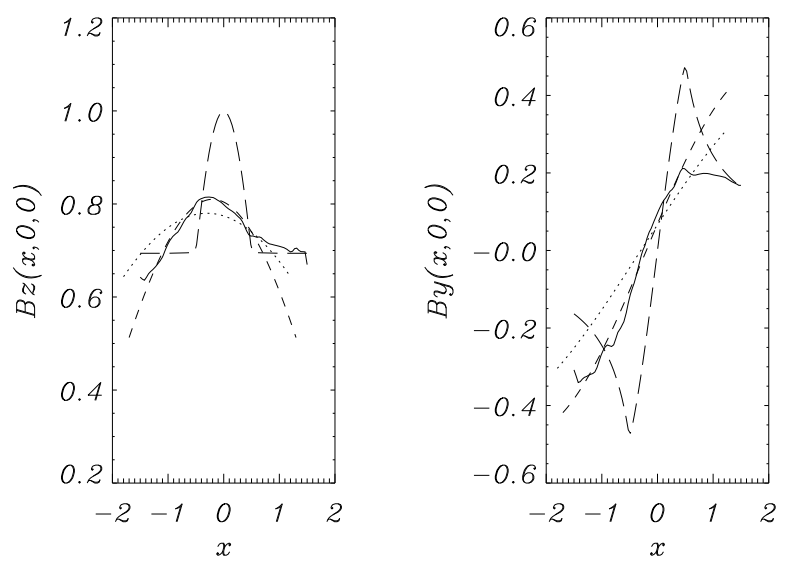

Fig. 9. Plots of $B_{z}(x, 0,0)($ left $)$ and $B_{y}(x, 0,0)$ for case (1) at $t=0$ (long dashed) and $t=400$ with results from the simulation (solid), predicted constant $\alpha$ (dotted) and best fit constant $\alpha$ short dashed).

is a remnant of the initial kink instability which displaces the axis in a helical manner. It is this displacement that has not yet relaxed. Note that the current profile in the final state still had spikes and is not completely flat (as indicated by the fairly high value of $j_{\max }$ shown in Fig. 8d) - but the fields are well fitted by a constant value of $\alpha$ which averages out the spikes as magnetic field is essentially an integral of the current.

It would be interesting to follow the time evolution further, but at present practical limitations due to an accumulation of numerical errors prohibit this. This will be a subject of future work.

\subsection{Case 2: $\alpha_{1}=3.0$ and $\alpha_{2}=-3.5$}

Case (2) is different from the others in that there is a region of reversed current surrounding the central core. The pitch of the helical instability of the loop is now in the opposite sense. The linear instability starts to develop around $t=80 \tau_{\mathrm{A}}$. The estimated initial growth-rate from the simulations, $\gamma=0.15$, differs 
Table 1. Predicted and numerical (fitted) values of $\delta W, \alpha$ and $B_{z}(0,0,0)$, and the offset of the cylindrical axis, for the 6 simulations.

\begin{tabular}{cccccccc}
\hline \hline Case & Pred. $\delta W$ & Actual $\delta W$ & Pred. $\alpha$ & Fit $\alpha$ & Pred. $B_{z}$ & Fit $B_{z}$ & Offset \\
\hline 1 & 1.7793 & 1.2804 & 0.5703 & 0.85 & 0.78 & 0.81 & -0.2 \\
2 & 9.0652 & 5.6677 & -0.7521 & -0.95 & 0.5954 & 0.659 & 0 \\
3 & 1.5859 & 1.2650 & 1.17 & 1.4 & 1.0086 & 1.05 & -0.1 \\
4 & 3.196 & 3.8446 & 2.4045 & 2.4 & 0.4708 & 0.456 & -0.47 \\
5 & 1.4034 & 0.9571 & 1.413 & 1.413 & 0.8075 & 0.8075 & -0.05 \\
6 & 1.4236 & 1.1243 & 1.6135 & 1.5135 & 0.7950 & 0.81 & -0.03 \\
\hline
\end{tabular}
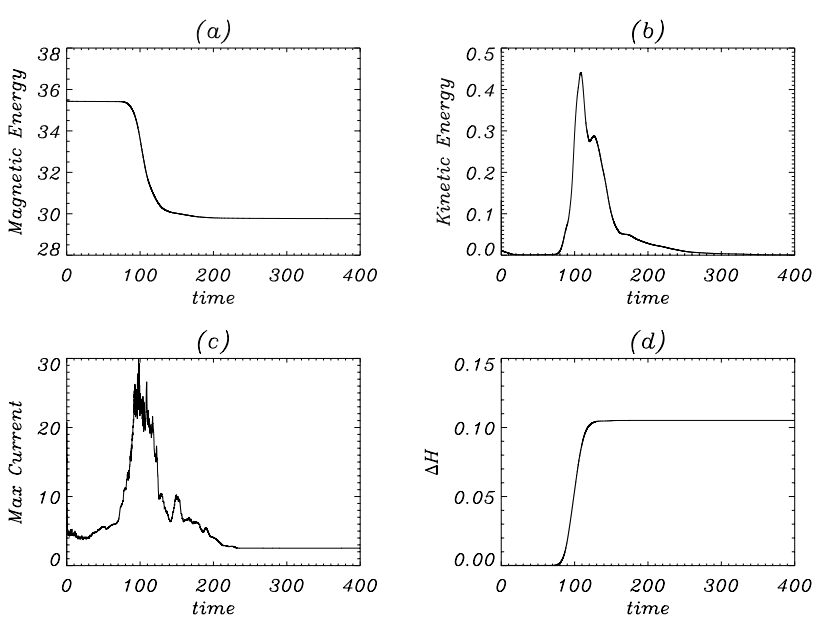

Fig. 10. Plots of magnetic energy (top, left hand side), kinetic energy (top, right hand side) internal energy (bottom, left hand side) and peak current (bottom, right hand side) for case (2).

quite significantly from the linear growth-rate from CILTS, as discussed in Sect. 4.7 below.

The magnetic energy drops by around 5.7 units - about $20 \%$ of the initial value - and then remains more or less constant; while the kinetic energy increases to a peak value around the time $t=110 \tau_{\mathrm{A}}$, before rapidly dropping down to a value below its initial value (Fig. 10). The release of a higher fraction of the initial magnetic energy is expected in this case, since the stronger variations in $\alpha$ are associated with increased free magnetic energy. After the instability and the current-sheet formation, the maximum current settles down to a value below the critical value. Hence, any subsequent evolution will rely on numerical diffusion to reach a new equilibrium. The helicity changes only evolves during the linear and nonlinear instability stage and stays at a constant value after the kinetic energy starts to decay. The final field profiles are well fitted with a constant- $\alpha$ force-free field, similarly to the method described for Case (1) above, and the fitted $\alpha$ value -0.95 is quite close to the predicted value of -0.7521 (see Table 1).

\subsection{Case 3: $\alpha_{1}=0.01$ and $\alpha_{2}=2.3$}

This equilibrium consists of a nearly untwisted central core, surrounded by a highly twisted sheath. This is the least unstable of the cases. In fact, whilst it indeed lies in the unstable region of Fig. 5, it would be stable if $R_{\mathrm{w}}$ were reduced to 1.5 (see Table 2) and hence is stable according to the original stability model with a conducting wall at the loop boundary (Fig. 1). This sensitivity to the wall postion may be due to the unusual shape of the most unstable mode (Fig. 4), which is not well-confined radially. The growth-rate of the instabiity is thus small (Table 2). A helical current-sheet has formed by $t=80 \tau_{\mathrm{A}}$, as shown in
Table 2. Predicted (linear stability) values and estimates from numerical simulations for growth-rate $\gamma$.

\begin{tabular}{cccc}
\hline \hline Case & Linear $\gamma\left(R_{\mathrm{W}}=3.0\right)$ & Linear $\gamma\left(R_{\mathrm{W}}=1.5\right)$ & Simulation $\gamma$ \\
\hline 1 & 0.049 & 0.047 & 0.06 \\
2 & 0.062 & 0.060 & 0.15 \\
3 & 0.048 & stable & 0.035 \\
4 & 0.202 & 0.194 & 0.15 \\
5 & 0.155 & 0.136 & 0.13 \\
6 & 0.180 & 0.152 & 0.15 \\
\hline
\end{tabular}

Fig. 11. Again this has the same structure as predicted by the linear instability calculations. However, the instability is not so clearly identified in the kinetic energy plot in Fig. 12b. In this case there are several periods when the kinetic energy grows almost exponentially. (The periods of exponential growth and the growth-rates are identified from a logarithmic plot, similar to Fig. 7.) The first case, from $t=25 \tau_{\mathrm{A}}$ to $45 \tau_{\mathrm{A}}$ has a growth-rate of about 0.035 . The linear growth-rate is 0.04776 . This mode appears to saturate and the kinetic energy drops before rising again in the time interval $[60,85]$, with a faster growth rate of 0.12 . The kinetic energy again saturates and drops before rising for a final time at $t=100 \tau_{\mathrm{A}}$ until $t=120 \tau_{\mathrm{A}}$. For this growth-period, the growth-rate is 0.0175 . Multiple kinetic energy peaks are observed for other cases, but not so strongly as here, and only this case has the first weak "precursor" peak in kinetic energy, apparently corresponding to the most unstable linear mode, followed by a much stronger peak. Figure 12 a shows that the magnetic energy drops during the instability by about 1.26 , but there appears to be a continual slow decay from $t=200 \tau_{\mathrm{A}}$ until the end of the simulation.

The maximum current (Fig. 12c) shows a sustained growth right from the start of the simulation (exceeding the critical value around $t=60 \tau_{\mathrm{A}}$ ) until the main peak in the kinetic energy is reached around $t=80 \tau_{\mathrm{A}}$. Although the maximum current starts to drop, it never falls below the critical value of 6.0 and so magnetic diffusion will continue till the end of the simulation.

Finally, we note that the change in the helicity is again negative. The helicity starts to change around $t=70 \tau_{\mathrm{A}}$ and is finished around $t=150 \tau_{\mathrm{A}}$. Again it is worth noting that the relative change in helicity is about $9 \times 10^{-4}$, while the relative change in magnetic energy is $2.4 \times 10^{-2}$. Hence, relaxation theory should give a reasonable description of the final state.

\subsection{Case 4: $\alpha_{1}=4.9$ and $\alpha_{2}=0.01$}

The fourth case is highly unstable compared with the first three, as confirmed by the re-calculated stability threshold (Fig. 5). In the original calculation without a potential-field layer, this equilibrium was close to the stable "tongue" region which projected into the unstable region, which was why it was chosen. The loop already shows signs of being kinked by $t=10 \tau_{\mathrm{A}}$ (Fig. 13) which is much quicker than for case (1) and the instability appears to 

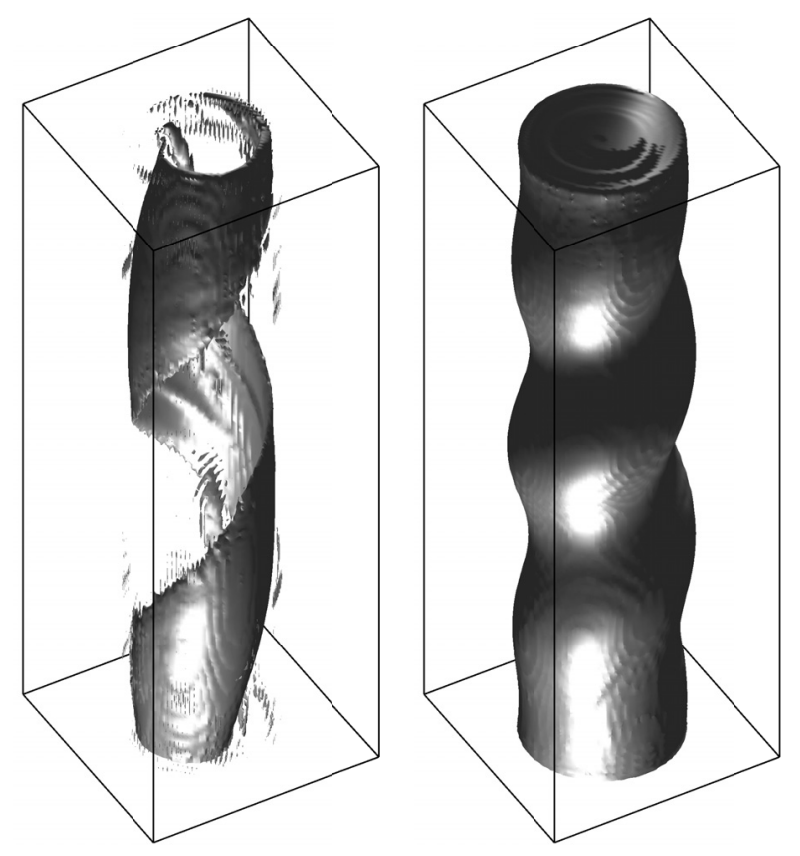

Fig. 11. Left: isosurfaces of the loop at $|j|=2.5$ at $t=80 \tau_{\mathrm{A}}$ for case (3). Right: isosurfaces of the loop at $|j|=0.75$ at $t=56 \tau_{\mathrm{A}}$ for case (5).

(a)

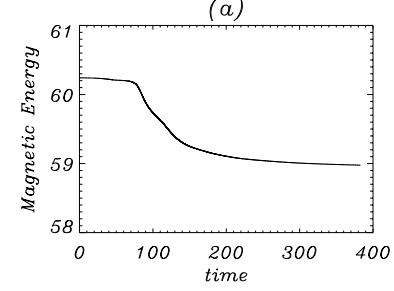

(c)

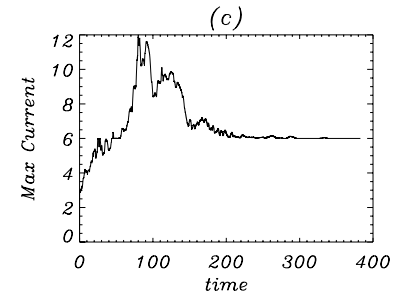

Fig. 12. Plots of magnetic energy (top, left hand side), kinetic energy (top, right hand side) internal energy (bottom, left hand side) and peak current (bottom, right hand side) for case (3). The initial helicity, calculated analytically, is 59.920 .

evolve faster. The estimated growth-rate is 0.15 , which again agrees well with the linear results $(\gamma \sim 0.2)$, and explains why the instability evolves much more rapidly than for case (1) where the growth-rate is three times slower.

This equilibrium has some features that are very different from those of the other equilibria investigated in this paper. A comparison of the isosurface shown in Fig. 13 with other isosurfaces in this paper reveals that it has a different structure from the others, with several kinks. This is consistent with the linear eigenfunction of this equilibrium as shown in Fig. 4. Note that both have 6 peaks in the axial direction (4 larger, and 2 smaller).

Another unique feature of this case is that the final state, when the field is close to being relaxed to equilibrium, has a significantly helical character (the cylindrical axis is displaced by 0.47 , see Table 1 ). This is in accordance with relaxation theory, which predicts that, if the initial helicity exceeds a critical
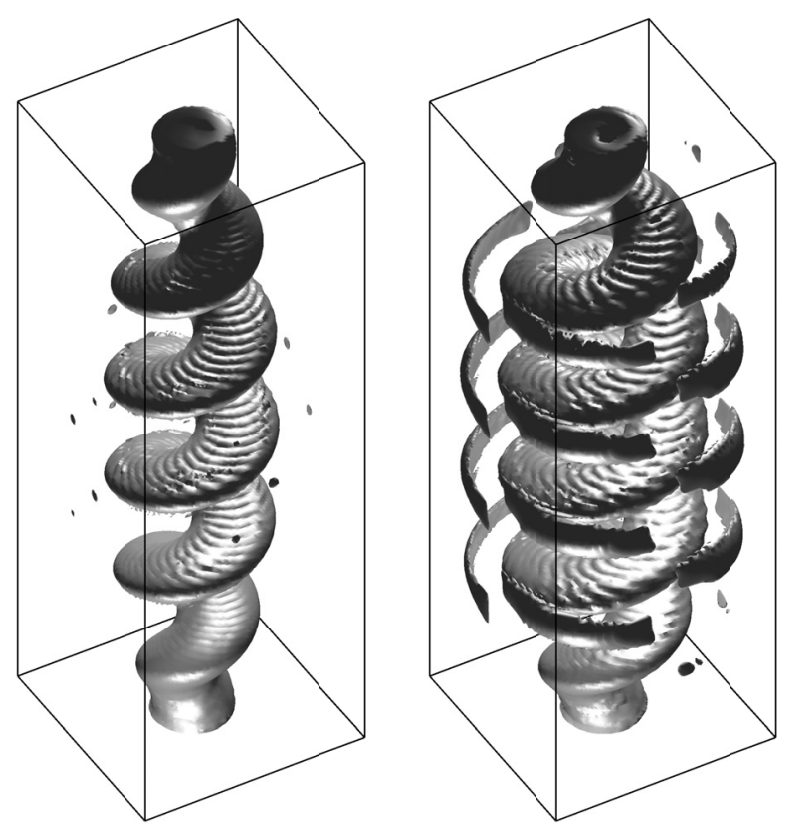

Fig. 13. Isosurfaces of the loop at $|j|=1.5$ at $t=24 \tau_{\mathrm{A}}$ (left hand side) and $t=32 \tau_{\mathrm{A}}$ (right hand side) for case (4).

value, the minimum-energy state is helical rather than axisymmetric (Taylor 1974; Browning \& Van der Linden 2003). The criterion can be expressed most simply in terms of the relaxed state $\alpha$ value, calculated assuming axisymmetry and normalised to the wall radius: if this exceeds 3.11 , the minimum-energy state is in fact helical. For case $4, \alpha * R_{\mathrm{w}}=3.608$ and hence a helical state is predicted. Note that the initial helicity is 4.822 , which seems to be low compared with the helicity of other cases. However, the more appropriately normalised helicity is $K / \psi_{\mathrm{w}}^{2}$ (evaluating the net axial flux $\psi_{\mathrm{w}}$ at the outer wall $R_{\mathrm{w}}$ ) which is larger than the other cases due to the low value of $\psi_{\mathrm{w}}=0.1710$. Relaxation theory would further predict that the normalised $\alpha$ for the relaxed helical state would be 3.11 (with any excess initial helicity increasing the magnitude of the helical distortion rather than increasing $\alpha$ ). The fitted value is somewhat larger than this (see Table 1); however, this has been calculated on the basis of fitting a shifted cylindrical model. Also, this field is not fully relaxed by the end of the simulation.

\subsection{Case 5: $\alpha_{1}=2.3$ and $\alpha_{2}=1.5$}

Cases (5) and (6) are just inside the upper boundary of the original marginal stability curve (Fig. 1), but are well inside the instability region when a potential field layer is included (Fig. 5). Case (5) becomes unstable by $t=50 \tau_{\mathrm{A}}$ (see Fig. 11) and the kink instability has a growth-rate of approximately 0.25 . During the non-linear phase of the instability a high current concentration builds up, starting to do so at $t=60 \tau_{\mathrm{A}}$. Again, this structure agrees well with the linear eigenfunction shown in Fig. 4.

\subsection{Case 6: $\alpha_{1}=2.5$ and $\alpha_{2}=1.69$}

Case (6) becomes noticeably kinked by $t=20 \tau_{\mathrm{A}}$ with a growthrate of approximately 0.14 ; the current isosurfaces are similar to Case (5).

The linear growth-rate, for $R_{\mathrm{W}}=3$, is 0.18 . A concentration of current begins to build up as a helical ribbon wrapped around the kinked central current at $t=60 \tau_{\mathrm{A}}$. 
The resistivity is triggered just after $t=60 \tau_{\mathrm{A}}$, when the critical current is reached. The magnetic energy drops a little and the kinetic energy increases and then peaks and begins to fall. The current plateaus while the cumulative ohmic heating and the helicity rise.

\subsection{Overview of cases 1 to 6}

Having seen the results for all six test cases, we now consider how the energy release and other quantities agree with the predictions of relaxation theory. This is given in Table 1 . which shows the predicted values of $\delta W, \alpha$ and the axial field strength, $B_{z}(0,0,0)$ based on relaxation theory and the actual change in magnetic energy and fitted values from the simulations. The offset (final column) represents the displacement of the cylindrical axis from the original axis. It should be noted, however, that only cases (1-3) have been run until relaxation is fairly complete. On the other hand, cases (4-6) are linearly more unstable and the kink instability develops much faster for them. For all cases, there is quite good agreement between the numerical results and relaxation theory, as summarised in Table 1. For the majority of cases, the numerical energy release is less or significantly less than that predicted by relaxation theory, which is entirely to be expected as the numerical simulations do not attain a fully relaxed state. Case (4) is unusal in that it releases slightly more energy than predicted. However, both the orders-of-magnitude and the trends are correct. There are also some other potential sources of small discrepancies: the geometries are slightly different (square boundary for numerics, cylinder for relaxation calculation); the numerical resolution of the simulation is quite coarse and the Lundquist number is not very high. Also, note that there is uncertainty in the correct choice of the boundary $\left(R_{\mathrm{w}}\right)$ for the relaxed state model: the relaxation may not develop over the whole volume within the external conducting boundary, but may be confined to a smaller region in the vicinity of the loop. This will lead to the actual energy release (numerical) being less than predicted, which holds for all cases except Case (4).

The results for the $\alpha$ values and the values of the axial field on the axis are summarised in Table 1 . Cases (4)-(6) are really very close to the values of $\alpha$ and $B_{z}(0,0,0)$ predicted by relaxation theory, with cases (1)-(3) still requiring some more relaxation. In addition, the best fit to the final state often is displaced by a small amount from the axis. This offset is listed in Table 1. The value of 0.03 in case (6) is close to the size of the grid and is probably not too significant. The offset is almost certainly left over from the kink instability that always displaces the magnetic axis in a helical manner and cannot be predicted from relaxation theory except when the values of $\alpha$ are so large that the relaxed state is no longer cylindrical but helical in nature. Only cases (1) and (4) show significant deviation from the initial state, suggesting that the final magnetic-field has a slight helical structure in these cases; as discussed above, this is in agreement with relaxation theory for case (4), which indeed has the largest offset.

The linear growth-rates can be compared with the estimated growth-rates calculated from the logarithm of the kinetic energy during the first initial rise of the instability and current sheet formation, as seen in Table 2, which shows predicted and numerical values of the growth-rate $\gamma$. However, in some cases the kinetic energy reaches a first maximum, drops and then rises a second and even third time. We do not use the growth-rates of these cases in this comparison. The numerical simulation were simply given an initial helical disturbance with a fairly localised radial extent. This, of course, does not match the most unstable mode and time is required for that mode to emerge. If there is more than one unstable mode, it may be that the fastest growth-rate is not correctly predicted. As discussed earlier, the square boundary of the numerical box lies in between circles of radii $R_{\mathrm{w}}=1.5$ and $R_{\mathrm{w}}=3$. Note that, in some cases, the linear growth-rate is affected by the choice of $R_{\mathrm{W}}$ within this range; these are the cases in which the eigenfunctions (Fig. 4) have signicant radial extent and are not confined to vicinity of the current-carrying loop. Case 2 seems to be somewhat anomalous, but in all other cases, the linear growth rate closely matches the estimated numerical value. All things considered, the agreement is actually very good.

\section{Discussion and conclusions}

The evolution of a linearly unstable twisted cylindrical forcefree flux tube, representing a coronal loop, has been investigated, focusing on the energy release. It has been proposed that coronal heating may result from a series of heating-events in which stored magnetic energy is released by magnetic reconnection - essentially the same process as in a solar flare. Previous work (Browning \& Van der Linden 2003) has proposed that such heating-events are triggered when the field becomes unstable to ideal MHD instabilities (kink instability in a simple loop geometry) and that the subsequent energy release in the nonlinear phase may be estimated by assuming a helicity-conserving relaxation to a minimum energy state. The model then predicts that heatingevents of a wide range of magnitudes may occur, depending on the current profile when the ideal stability threshold is crossed. Here, we have explored some aspects of this model using a 3D MHD simulation.

A coronal loop is modelled as a straight cylinder of twisted magnetic-field, embedded in a potential field layer and (for numerical purposes) an outer conducting wall at a large radius. The magnetic field lines are line-tied at the ends of the cylinder, representing the photosphere. The initial current profile is parameterised according to the variation in $\alpha$ (the normalised ratio of current to magnetic field), whereby $\alpha$ is piece-wise constant. Six test cases were studied, with a range of current profiles, all of which are unstable force-free equilibria. Three cases were chosen that lay close to the marginal stability curve, when a conducting wall surrounds the loop. On relaxing this, by placing a potential field around the loop and with the outer boundary sufficiently far away that the growth rate is not influenced, these cases turned out to be well within the unstable region, having large growth-rates. The other three cases were located close to, but just outside, the newly calculated marginal stability curve, having relatively small growth-rates.

In all cases, a kink instability is initially excited; subsequently a helical current sheet wraps itself around the loop, whose shape is very well predicted by the eigenfunction of the linear instability. The initial growth-rate of instability agrees very well with the predictions of the linear stability code. In the nonlinear development, the strength of the current in the sheet exceeds a critical value, triggering anomolous resistivity and fast magnetic reconnection. The reconnection allows the magneticfield to change its topology and relax towards its lowest energy state, conserving axial flux and almost conserving magnetic helicity as it evolves. The relative change in helicity is always about one hundredth of the relative change in magnetic energy, indicating that relaxation theory will apply. (This ratio is constrained by the grid-size - we would expect even better conservation of magnetic helicity on finer grids, as will be tested in future work.) All cases relax towards a constant- $\alpha$ state (though not quite down to the predicted one). The $\alpha$ profile is not constant but does vary 
rapidly about a mean value and it is the mean value that gives the best fit to the axial and azimuthal field profiles at the end of the simulation. However, despite the remaining "spikiness" in the $\alpha$ profile, the magnetic-field profiles and the magnetic energy are very close to those of the constant $\alpha$ state. It is likely that in subsequent evolution, the current profile will be smoothed further.

The numerical simulations confirm many of the key predictions of the Browning \& Van der Linden (2003) coronal heating model: in particular, as outlined above, the development of the kink instability, the onset of reconnection leading to magnetic energy release, the conservation of helicity, the evolution to a relaxed state which is (close to) a linear force-free field. The initial stages of evolution agree very well with the predictions of the linear instability calculations. The release in magnetic energy agrees reasonably well with the predicted values. Also, the relaxed state is found to be helical in some cases, in agreement with relaxation theory. Most importantly, the numerical results confirm the central prediction that heating events of a wide range of magnitudes will occur, depending on where the stability boundary is crossed. Within this theory, coronal heating by nanoflares arises naturally, and the energy distribution of nanoflares can be predicted. The coronal field will evolve in a way which results in sporadic heating: the field is stressed until it becomes unstable and then relaxes, releasing stored magnetic energy, and then the process repeats, leading to a sequence of discrete heating-events of varying magnitude.

The discrepancies between the simulation results and relaxation theory are also interesting, and the simulations also eluciduate the dynamic process by which energy is released, which is beyond the scope of relaxation theory. It appears that relaxation is most effective in fields which are strongly unstable. Also, the presence of multiple unstable modes seems to contribute to the establishment of a relaxed state. It is not clear why in some cases the final state is well fitted by a constant- $\alpha$ force free field but with a value of $\alpha$ differing significantly from predictions. The energy release due to relaxation is strongly dependent of the extent of the region over which relaxation occurs: the numerical simulations show that this depends on the nonlinear amplitude of the kink instability, which differs for different profiles. Some interesting features are apparent in the nonlinear dynamics, such as successive phases of exponentially growing kinetic energy. Further investigation of this nonlinear dynamical behaviour, and the nature of the magnetic reconnection, will be a subject of future work.

Whilst the simulations are suggestive of reconnection processes which might occur in coronal loops, and hence heat the corona, a direct quantitative comparison in terms of small scale processes cannot be made because our simulation - in common with all other simulations of the solar corona - has a far lower value of the Lundquist number and has a very simplified model of "anomalous resistivity" through Eq. (15). Also, of course, kinetic effects, which are likely to be significant at small scales within the corona, are neglected. Dissipation in our code occurs mainly through anomalous resistivity, though viscous dissipation of kinetic energy also plays a role. However, it is very likely that the global dynamics and the overall energy release is only very weakly dependent on the details of the localised dissipative processes; this will be investigated further in future work through scaling studies. Thus, the general scenario of current sheet generation leading to magnetic reconnection and energy dissipation should be applicable to the corona, even if the details within the current sheets are not.
The results have significant implications for understanding solar coronal heating. Firstly, we confirm the key finding of Browning \& Van der Linden (2003): even within a single loop structure, heating events with a wide distribution of magnitudes can occur (with the magnitude of the energy release depending on the point at which the marginal stability curve is crossed, and hence on the time history of the footpoint twisting.) Heating will naturally be sporadic and time-dependent, with a series of energy releasing events of varying sizes. This provides theoretical backing for the nanoflare scenario proposed by Parker 1988. The energy release quantum (elemental nanoflare) is

$$
\frac{R^{3} B^{2}}{\mu_{0}} \delta W,
$$

where $\delta W$ is the smallest dimensionless energy release from Table 1 . Taking typical active region values of $B=0.01 \mathrm{~T}$ and $R=1 \mathrm{Mm}$ gives an elemental nanoflare of about $8 \times 10^{19} \mathrm{~J}$ or less, consistent with Parker's ideas.

Our model does not directly predict a coronal heating rate, as it calculates only energy release from isolated events. Such events will repeat, and the time-averaged heating will depend on the rate at which the external driving triggers crossings of the stability threshold. In terms of an order of magnitude heating rate, our model is similar to other reconnection-based coronal heating models: since free energy cannot build up significantly beyond the stability threshold, the heating rate will be given by the Poynting flux $(P)$ into the corona. Following Parker (1983), this is

$P=\frac{B_{\mathrm{t}} B_{\mathrm{v}} v_{\text {phot }}}{\mu_{0}}$

as is well known, typical coronal values of the vertical magnetic field $\left(B_{v}=0.01 \mathrm{~T}\right)$ and the photospheric velocity $\left(v_{\text {phot }}=\right.$ $1 \mathrm{~km} \mathrm{~s}^{-1}$ ) give a sufficient energy flux for active region heating $\left(\approx 10^{4} \mathrm{~W} \mathrm{~m}^{-2}\right.$ ) so long as the transverse (horizontal) magnetic field component $\left(B_{\mathrm{t}}\right)$ is high enough. Allowing for $25 \%$ of free energy to be dissipated, Parker (1983) states the requirement $B_{\mathrm{t}} \approx 0.4 B_{v}$. This leads to the idea that for coronal heating by reconnection to be effective, there must be some control mechanism maintaining a twist or shear at this critical level: reconnection must only "switch-on" when this degree of shear is reached (Dahlburg et al. 2005). Thus, a second significant implication of our results for coronal heating is that we provide some explanation for this switch-on nature of reconnection, since heating occurs only when the kink instability threshold is reached, which requires a critical $B_{\theta} / B_{z}$. The value of this ratio varies between cases; clearly, instability does not occur until this is sufficiently large, and typical values are in the range $0.1-1$.

Acknowledgements. The computational work was carried out on the joint STFC and SFC (SRIF) funded linux clusters at St Andrews University. P.K.B. is grateful to UK PPARC/STFC for financial support.

\section{References}

Arber, T. D., Longbottom, A. W., \& Van der Linden, R. A. M. 1999, ApJ, 517, 990

Arber, T. D., Longbottom, A. W., Gerrard, C. L., \& Milne, A. M. 2001, J. Comput. Phys., 171, 151

Aschwanden, M. J. 1999, Sol. Phys., 190, 233

Aschwanden, M. J., \& Parnell, C. E. 2002, ApJ, 572, 1048

Baty, H., \& Heyvaerts, J. 1996, A\&A, 308, 935

Baty, H. 2000, A\&A, 360, 345

Baty, H. 2001, A\&A, 367, 321 
Benz, A. O., \& Krucker, S. 2002, ApJ, 568, 413

Berger, M. A. 1984, Geophys. Astrophys. Fluid Dyn., 30, 79

Browning, P.K. 1988, J. Plasma Phys., 40, 263

Browning, P. K. 1991, Plasma Phys. Control. Fusion, 33, 359

Browning, P. K., \& Priest, E. R. 1986, A\&A, 159, 129

Browning, P. K., Sakurai, T., \& Priest, E. R. 1986, A\&A, 158, 217

Browning, P. K., \& Van der Linden, R. A. M. 2003, A\&A, 400, 355

Dahlburg, R. B., Klimchuck, J. A. \& Antiochos, S. K. 2005, ApJ, 622, 1191

De Bruyne, P., \& Hood, A. W. 1992, Sol. Phys., 142, 87

Evans, C. R., \& Hawley, J. F. 1988, ApJ, 332, 659

Finn, J. M., \& Antonsen, T. A. 1985, Communications in Plasma Physics and Controlled Fusion 9, 111

Heyvaerts, J., \& Priest, E. R. 1984, A\&A, 137, 63

Ji, H. 1998, in Magnetic Helicity in Space and Laboratory Plasmas, Geophys. Monograph 111, ed. M. R. Brown, R. C. Canfield, \& A. A. Pevtsov, 141 Klimchuck, J. A. 2006, Sol. Phys., 234, 41

Kusano, K., \& Nishikawa, K. 1996, ApJ, 461, 415

Lothian, R. M., \& Browning, P. K. 2000, Sol. Phys., 194, 205
Mandrini, C. H., Demoulin, P., \& Klimchuck, J. 2000, ApJ, 530, 999

Melrose, D. B. 2004, Sol. Phys., 221, 121

Melrose, D. B., Nicholls, J., \& Broderick, N. G. 1994, J. Plasma Phys. 51, 163

Nandy, D., Hahn, M., Canfield, R. C., \& Longcope, D. W. 2003, ApJ, 597, L73

Narain, U., \& Ulmschneider, P. 1996, Space Sci. Rev., 75, 453

Parker, E. N. 1983, ApJ, 264, 642

Parker, E. N. 1988, ApJ, 330, 474

Parnell, C. E., \& Jupp, P. E. 2000, ApJ, 529, 554

Priest, E. R., \& Forbes, T. G. 2002, A\&ARv, 10, 313

Priest, E. R., Longcope, D. W., \& Heyvaerts, J. 2005, ApJ, 624, 1057

Taylor, J. B. 1974, Phys. Rev. Lett., 33, 1139

Taylor, J. B. 1986, Rev. Mod. Phys., 58, 741

Van der Linden, R. A. M., \& Hood, A. W. 1999, A\&A, 346, 303.

Van Leer, B. 1997, J. Comput. Phys., 135, 229

Vekstein, G. E., Priest, E. R., \& Steele, C. D. C. 1993, ApJ, 417, 781

Velli, M., Lionello, R., \& Einaudi, G. 1997, Sol. Phys., 172, 257

Zhang, M., \& Low, B. C. 2003, ApJ, 584, 479 